\title{
Effect of the Target Deformation on Incomplete Fusion Dynamics
}

\author{
D. Singh ${ }^{1,}$, Rahbar Ali ${ }^{2}$, M. Afzal Ansari ${ }^{3}$, R. Kumar ${ }^{4}$, R. P. Singh ${ }^{4}$, S. Muralither ${ }^{4}$ and R. K. Bhowmik ${ }^{4}$ \\ ${ }^{1}$ Centre for Applied Physics, Central University of Jharkhand, Ranchi-835 205, India \\ ${ }^{2}$ Department of Physics, G. F. (P. G.) College, Shahjahanpur-242 001, India \\ ${ }^{3}$ Department of Physics, Aligarh Muslim University, Aligarh-202 002, India \\ ${ }^{4}$ Inter University Accelerator Centre, Aruna Asaf Ali Marg, New Delhi-110 067
}

\begin{abstract}
To investigate the role of target deformation on incomplete fusion dynamics, a particle-gamma coincidence experiment has been performed at Inter University Accelerator Centre, New Delhi. Spin distributions for various evaporation residues populated via complete and incomplete fusion of ${ }^{16} \mathrm{O}$ with ${ }^{124} \mathrm{Sn}$ at $6.3 \mathrm{MeV} /$ nucleon have been measured. Experimentally measured spin distributions of the residues produced as incomplete fusion products associated with fast $\alpha$ and $2 \alpha$-emission channels observed in forward cone are found to be distinctly different from those of the residues produced as complete fusion products. The mean value of input angular momentum $\mathrm{J}_{0}$ for evaporation residues produced through $\mathrm{xn}$ channels (complete fusion products) is found to be $\mathrm{J}_{0} \approx 7 \mathrm{~h}$, while the mean value of input angular momentum $\mathrm{J}_{0}$ for the residues produced through direct $\alpha \times n$ and $2 \alpha \times n$ channels (incomplete fusion products) in forward cone, are found to be $\mathrm{J}_{0} \approx 9 \hbar$ and $\approx 12 \hbar$ respectively for ${ }^{16} \mathrm{O}+{ }^{124} \mathrm{Sn}$ (spherical) system [7]. The mean value of input angular momentum $\mathrm{J}_{0}$ for the system ${ }^{16} \mathrm{O}+{ }^{169} \mathrm{Tm}$ (deformed) reported in ref. [8], are found to be $\approx 10 \hbar$ for xn-channels (complete fusion products) and for direct $\alpha \times n$ and $2 \alpha x n$ channels (incomplete fusion products) the value of $\mathrm{J}_{0}$ approaches to $\approx 13 \hbar$ and $\approx 16 \hbar$, respectively. The mean values of the input angular momentum observed for xn (complete fusion products), $\alpha \times n$ and $2 \alpha \times n$ (incomplete fusion products) in ${ }^{16} \mathrm{O}+{ }^{124} \mathrm{Sn}$ (spherical) system are smaller than that of the mean values of the input angular momentum observed for xn (complete fusion products), $\alpha x n$ and $2 \alpha \times n$ (incomplete fusion products) in ${ }^{16} \mathrm{O}+{ }^{169} \mathrm{Tm}$ (deformed) system. The comparison of data inferred that the mean values of the input angular momentum are smaller in case of spherical target than that of deformed target at same projectile energy of ${ }^{16} \mathrm{O}$-ion beam. It means that the target deformation affect the incomplete fusion dynamics.
\end{abstract}

\section{Introduction}

The study of incomplete fusion (ICF) dynamics induced by heavy ions has been a growing interest at energies above Coulomb barrier. The first experimental evidence of ICF reactions was given by Britt and Quinton [1], who observed the break-up of the incident projectiles like ${ }^{12} \mathrm{C},{ }^{14} \mathrm{~N}$ and ${ }^{16} \mathrm{O}$ into alpha clusters in an interaction with the surface of the target nucleus at bombarding energies $\approx 10.5 \mathrm{MeV} /$ nucleon. Subsequently, Galin et al. [2] also observed the break-up of projectile and called such reactions, leading to the emission of "fast" alpha particles, as 'ICF reaction' or 'break-up fusion reaction'. The major advances in the understanding of ICF dynamics were made after the charged particle- $\gamma$ coincidence measurements by Inamura et al. [3]. Some recent observations [4-6] show that at projectile energies above the Coulomb barrier, both the processes complete fusion (CF) and incomplete fusion (ICF) may be considered as dominant reaction mechanisms. Semiclassical theory of heavy ion interaction says that the $\mathrm{CF}$ and ICF processes may be categorized on the basis of input angular momentum imparted in the system. In $\mathrm{CF}$, the attractive nuclear potential overcomes the repulsive Coulomb and centrifugal potentials in central and near central collisions. Consequently, CF takes place at a small value of impact parameter, where as the formation of fully equilibrated compound nucleus takes place. However, at relatively higher values of impact parameter, the repulsive centrifugal potential increases and hence the dominance of attractive nuclear potential ceases to capture entire projectile. Therefore, an incompletely fused composite system comprising of a part of projectile plus the target appears in the exit channel that leads to ICF process, wherein the involvement of input angular momentum is relatively larger than that of needed for $\mathrm{CF}$ process to take place. At this stage if the input angular momentum exceeds the critical limit for $\mathrm{CF}$, no fusion

\footnotetext{
$\bar{a}$ Corresponding author: dsinghiuac@gmail.com
} 
can occur unless a part of the projectile is emitted to release excess input angular momentum. As such, prompt emission of a part of projectile takes place to provide sustainable input angular momentum to the system. After such an emission, the resulting input angular momentum carried by the remnant projectile is less than or equal to its own critical angular limit for fusion to occur with the target nucleus. Various dynamical models viz. Break-Up Fusion (BUF) [7], Sumrule [8], Promptly Emitted Particles (PEPs) [9], Hot Spot [10], Multistep Direct Reaction theory [10] etc. have been proposed to explain the mechanism of ICF reactions. However, no theoretical model is available to explain the gross features of experimental data available below $\mathrm{E} / \mathrm{A}=10 \mathrm{MeV}$. Most of the ICF dynamics studies by charged particle-gamma coincidence technique have been carried out with low- $Z$ $(Z \leq 10)$ projectile induced reactions on heavy targets $(\mathrm{A} \geq 150)$. However, with medium mass target nuclei, such information's are scarce. An attempt has been made to investigate the role of target deformation on ICF dynamics. Spin distributions for various evaporation residues populated via complete and incomplete fusion of ${ }^{16} \mathrm{O}$ with ${ }^{124} \mathrm{Sn}$ at $6.3 \mathrm{MeV} /$ nucleon have been measured. The measured mean values of input angular momentum in ${ }^{16} \mathrm{O}+{ }^{124} \mathrm{Sn}$ (spherical) system [11] have been compared with the mean value of input angular momentum in ${ }^{16} \mathrm{O}+{ }^{169} \mathrm{Tm}$ (deformed) system [12].

\section{Experimental details}

The present in-beam charged-particle gamma coincidence experiment was performed at the Inter-University Accelerator Centre (IUAC), New Delhi, India, using the15UD Pelletron Accelerator. A self-supporting target of ${ }^{124} \mathrm{Sn}$ (enrichment $\approx 97.20 \%$ ) of thickness $2 \mathrm{mg} / \mathrm{cm}^{2}$ were prepared by rolling machine. The $\gamma$-detector array (GDA) consists of 12 Compton-suppressed HPGe detectors at angles of $45^{\circ}, 99^{\circ}$ and $153^{\circ}$ with respect to the beam direction. Four HPGe detectors arranged at each of these angles along with a charged-particle-detector array (CPDA) consisting of 14 Phoswich detectors housed in a small scattering chamber arranged in two truncated hexagonal pyramids. All 14 detectors of the CPDA are divided into three angular zones. There are 4 detectors at "forward angles" (F) $\left(10^{0}-60^{0}\right), 4$ detectors at "backward angles" (B) $\left(120^{\circ}-170^{\circ}\right)$, and 6 detectors at "sideways angles (S), $\left(60^{\circ}-120^{\circ}\right)$. The energy calibration of the Compton suppressed HPGe detectors and CPDs were done by using ${ }^{152} \mathrm{Eu} \gamma$-ray and ${ }^{241} \mathrm{Am} \alpha$-sources of known strength. In the present experiment, two groups of $\alpha$ particle are detected by forward angle CPDs: (i) the fusion-evaporation (CF) $\alpha$-particles of average energy $\mathrm{E}_{\alpha}-\mathrm{CF} \approx 17 \mathrm{MeV}$ and (ii) the ICF fast $\alpha$-particles of energy $\mathrm{E}_{\alpha}-\mathrm{ICF} \approx 25 \mathrm{MeV}$. The $\alpha$-particles emitted during the break up of ${ }^{16} \mathrm{O}$ - ion beam and moves in the forward cone. The energy and velocity of these $\alpha$-particles will larger than those of $\alpha$-particles emitted from compound nucleus. These $\alpha$-particles are called "fast" $\alpha$-particles. In front of the each four "forward-cone" CPDs, aluminium absorbers of appropriate thickness were used to stop "evaporation" $\alpha$-particles $\left(\mathrm{E}_{\alpha}-\mathrm{ICF} \approx 17 \mathrm{MeV}\right)$.
Hence, only fast or ICF $\alpha$ particles with energy greater than $17 \mathrm{MeV}$ have been detected in the forward cone.

\section{Analysis of the experimental data}

The off-line data analysis has been carried out using software INGASORT [13]. Projecting four coincidence gating conditions like particle-forward, particlebackward, $\alpha$-forward, and $\alpha$-backward on all the observed $\gamma$-ray spectra and identification of specific $\mathrm{CF}$ and ICF reaction products were done using forward and backward $\alpha$-gated spectra. Spin distributions for different evaporation residues have been extracted by measuring the relative production yields of different levels in the rotational bands. In other words, in this experiment feeding patterns of the rotational levels of the evaporation residues populated by $\mathrm{CF}$ and ICF processes have been studied by measuring the relative yields of the rotational levels from the intensities of gamma transitions for the evaporation residues using the particle-gated spectra under the above mentioned gating conditions. Gamma ray energies and their intensities used in the present work have been taken from RADWARE level scheme directory [14].

\section{Results and Discussion}

The ICF reaction channels that were identified in the forward cone in coincidence with fast $\alpha$-particles are ${ }^{124} \mathrm{Sn}(\mathrm{O}, \alpha 3 \mathrm{n}) \quad{ }^{133} \mathrm{Ba}, \quad{ }^{124} \mathrm{Sn}(\mathrm{O}, \alpha 4 \mathrm{n}) \quad{ }^{132} \mathrm{Ba}$, ${ }^{124} \mathrm{Sn}(\mathrm{O}, \alpha 5 \mathrm{n}) \quad{ }^{131} \mathrm{Ba}, \quad{ }^{124} \mathrm{Sn}(\mathrm{O}, \alpha \mathrm{p} 4 \mathrm{n}) \quad{ }^{131} \mathrm{Cs}$, ${ }^{124} \mathrm{Sn}(\mathrm{O}, \alpha 2 \mathrm{pn}) \quad{ }^{133} \mathrm{Xe}, \quad{ }^{124} \mathrm{Sn}(\mathrm{O}, \quad 2 \alpha 2 \mathrm{n}){ }^{130} \mathrm{Xe}$, ${ }^{124} \mathrm{Sn}(\mathrm{O}, 2 \alpha 4 \mathrm{n}){ }^{128} \mathrm{Xe}$ and $\quad{ }^{124} \mathrm{Sn}(\mathrm{O}, 2 \alpha \mathrm{p} 4 \mathrm{n}){ }^{127} \mathrm{I}$. The reaction channels which have been identified in backward direction are ${ }^{124} \mathrm{Sn}(\mathrm{O}, \mathrm{p} 4 \mathrm{n}){ }^{135} \mathrm{La},{ }^{124} \mathrm{Sn}(\mathrm{O}, \mathrm{p} 6 \mathrm{n}){ }^{133} \mathrm{La}$, ${ }^{124} \mathrm{Sn}(\mathrm{O}, \alpha 3 \mathrm{n}){ }^{133} \mathrm{Ba}, \quad{ }^{124} \mathrm{Sn}(\mathrm{O}, \alpha 4 \mathrm{n}){ }^{132} \mathrm{Ba}$, ${ }^{124} \mathrm{Sn}(\mathrm{O}, \alpha 5 \mathrm{n}){ }^{131} \mathrm{Ba}, \quad{ }^{124} \mathrm{Sn}(\mathrm{O}, \quad 2 \alpha 2 \mathrm{n}){ }^{130} \mathrm{Xe} \quad$ and ${ }^{124} \mathrm{Sn}(\mathrm{O}, \alpha \mathrm{p} 4 \mathrm{n}) \quad{ }^{131} \mathrm{Cs}$. The neutron channel ${ }^{124} \mathrm{Sn}(\mathrm{O}, 7 \mathrm{n}){ }^{133} \mathrm{Ce},{ }^{124} \mathrm{Sn}(\mathrm{O}, 6 \mathrm{n}){ }^{134} \mathrm{Ce},{ }^{124} \mathrm{Sn}(\mathrm{O}, 5 \mathrm{n}){ }^{135} \mathrm{Ce}$ has also been identified from the singles spectra and confirmed from decay gamma lines. The CF and ICF channels can also be identified by comparing the spectra of forward and backward going alpha particles. ICF would thus lead to a 'harder' alpha spectrum in the forward direction than expected for alpha evaporation from a compound nucleus. The measured spin distribution produced in $\mathrm{CF}$ (fusion-evaporation) channels are displayed in Fig. 1(a)-(d). The spin distributions are the relative yield profile as a function of observed spin of evaporation residues. The dotted lines represent the spin at which the relative yield is half of the ERs produced through CF and ICF. The spins at which the relative yield is half, it means that the relative yield is the average relative yield. The angular momentum corresponding to this relative yield is called as the average angular momentum. The spin distributions show a sharp exponential fall in the intensities of cascade $\gamma$ transitions with spin of the states and give an indication 
of strong side-feeding to the lowest member of the yrast line transitions. It is worth to note that $\alpha$-particles are emitted in backward direction i.e. CF $\alpha$-particles. It can be seen clearly from the Figs. 1(b)-(d) that the spin distribution plots of the residues ${ }^{133} \mathrm{Ba},{ }^{132} \mathrm{Ba},{ }^{131} \mathrm{Ba}$, ${ }^{130} \mathrm{Xe},{ }^{131} \mathrm{Cs}$ and ${ }^{127} \mathrm{I}$, identified from backward $\alpha$-gated spectra, are found to be distinctly different from those identified from (ICF) forward $\alpha$-gated spectra. The spin distributions of the above mentioned residues identified from backward $\alpha$-gated spectra follow the similar trend as that has been observed in $\mathrm{CF}$ channels (i.e. in $x n$ and pxn channels), displayed in Fig.1 (a). The measured relative production yields of the

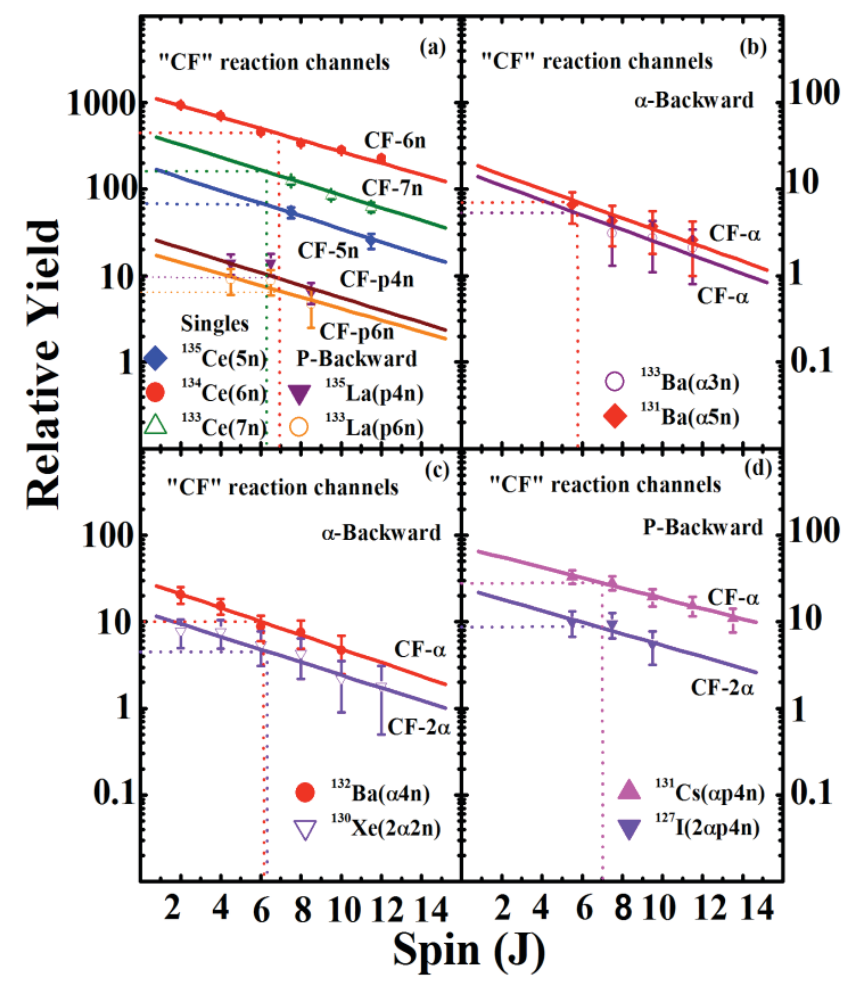

Figure 1. (Colour online) (a)-(d) experimentally measured spin distribution of evaporation residues produced in $\mathrm{CF}$ for the $\operatorname{system}^{16} \mathrm{O}+{ }^{124} \mathrm{Sn}$ at 6.3 $\mathrm{MeV} /$ nucleon. The straight lines are the least-squares fits.

above ICF evaporation residues, observed in coincidence with fast $\alpha$-particle(s) emitted in forward cone, are shown in Fig. 2(a)-(c). The spin distribution curves in this Figs.2 (a)-(c) show a least square fit to the measured relative production yields of these evaporation residues, using a function of the type:

$$
Y=Y_{0} /\left[1+\exp \left(J-J_{0}\right) / \Delta\right]
$$

where $\Delta$ is related to the width of mean input angular momentum $J_{0}$, and $Y_{0}$ is the normalization constant. Here $J_{0}$ is a sensitive parameter, which provides the qualitative information about the input angular momentum $\ell$ associated with various reaction channels.
The relative production yields for evaporation residues ${ }^{133} \mathrm{Ba},{ }^{132} \mathrm{Ba},{ }^{131} \mathrm{Ba},{ }^{131} \mathrm{Cs}$ and ${ }^{133} \mathrm{Xe}$ are found to be constant up to spin $\mathrm{J} \approx 7 \mathrm{~h}$ and for evaporation residues ${ }^{130} \mathrm{Xe},{ }^{128} \mathrm{Xe}$ and ${ }^{127} \mathrm{I}$ constant up to spin $\mathrm{J} \approx 9 \hbar$ respectively and then successively decreases exponentially with higher spin states, indicating hereby the absence of side-feeding to the lower members of yrast line transitions. It has been observed from these figures that the spin at half-yield for fast $\alpha$ and $2 \alpha$-emitting reaction channels (associated with ICF), observed in forward cone, comes out to be $\mathrm{J}_{0} \approx 9 \hbar$ and $\approx 12 \hbar$ respectively. The spin at half-yield $\mathrm{J}_{0}$ for these
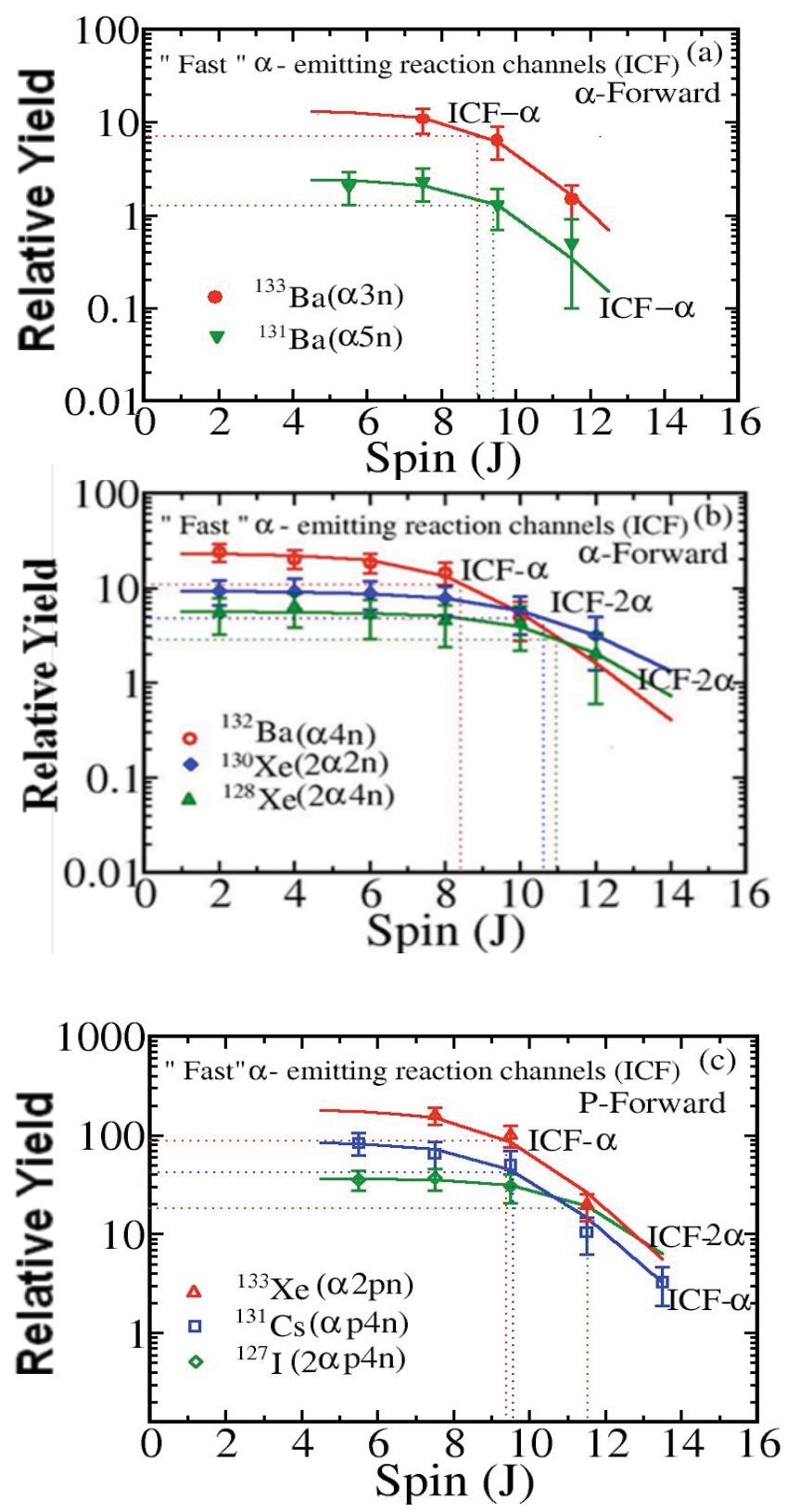

Figure 2. (Colour online) (a)-(c) Experimentally measured spin distribution of evaporation residues produced in ICF for the system ${ }^{16} \mathrm{O}+{ }^{124} \mathrm{Sn}$ at 6.3 $\mathrm{MeV} /$ nucleon. The curves through experimental points are the best fits using the expression (1). 
evaporation residues produced through $\mathrm{CF}$ reaction channels is found to be $\mathrm{J}_{0} \approx 7 \mathrm{~h}$, while the spin at half-yield $\mathrm{J}_{0}$ for the residues produced through ICF reaction channels in fast $\alpha$ and $2 \alpha$-emission in forward cone, was found to be $\mathrm{J}_{0} \approx 9 \hbar$ and $\approx 12 \hbar$ respectively. The mean value of input angular momentum $\mathrm{J}_{0}$ is reported in Ref. [8], for the system ${ }^{16} \mathrm{O}+{ }^{169} \mathrm{Tm}$ (deformed) are found to be $\approx 10 \mathrm{~h}$ for $\mathrm{xn}$-channels (CF products) and for direct $\alpha \times n$ and $2 \alpha \times n$ channels (ICF products) the value of $\mathrm{J}_{0}$ approaches to $\approx 13 \hbar$ and $\approx 16 \hbar$, respectively.

The accuracy of the presently measured relative yields has been checked by comparing the measured one with theoretically calculated relative yields using theoretical model code PACE2. The measured relative yields of the individual reaction products produced in (CF) 'fusion-evaporation' channels has been extrapolated up to $J=0 \hbar$ and then has been normalized with the total yield by taking sum of all 'fusion-evaporation' ( $\mathrm{xn} / \mathrm{pxn}$ ) channels. On the other hand relative yield of individual reaction products calculated using PACE2 has been normalized with the total yield of 'fusion-evaporation' channels. The yield ratio $\left(Y_{E X P} / Y_{P A C E 2}\right)$ for all 'fusionevaporation' channels has been plotted in Fig.3. It has

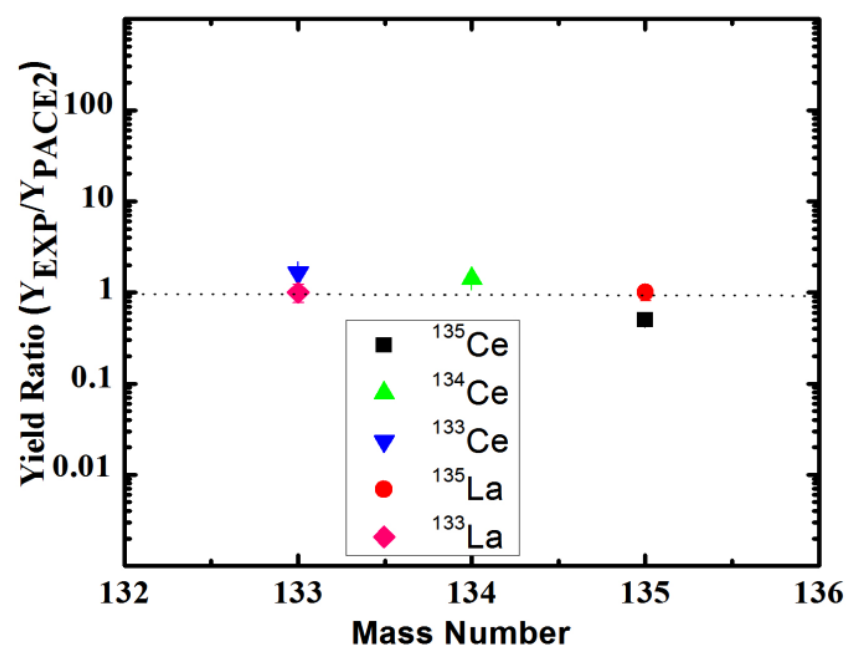

Figure 3. (Colour online) The ratio of experimentally measured and theoretically estimated relative production yields of the residues populated via $\mathrm{CF}$ in $\mathrm{xn}$ and $\mathrm{pxn}$ channels for the system ${ }^{16} \mathrm{O}+{ }^{124} \mathrm{Sn}$ at $100 \mathrm{MeV}$ energy.

been observed that the experimental and theoretical values are close to each other and hence the correctness of measured relative yields has been established.

It can be seen clearly from the above results, the mean values of the input angular momentum observed for $\mathrm{xn}$ (CF products), $\alpha \mathrm{xn}$ and $2 \alpha \mathrm{xn}$ (ICF products) in ${ }^{16} \mathrm{O}+{ }^{124} \mathrm{Sn}$ (spherical) system are smaller than that of mean values of input angular momentum observed for $\mathrm{xn}$
(CF products), $\alpha \times n$ and $2 \alpha \times n$ (ICF products) in ${ }^{16} \mathrm{O}+{ }^{169} \mathrm{Tm}$ (deformed) system. The comparison of these results inferred that the mean values of input angular momenta are smaller in case of spherical target than that of deformed target at same projectile energy of ${ }^{16} \mathrm{O}$-ion beam. It means that the target deformation affect the ICF dynamics. These results also suggest that for the study of role of target deformation on ICF dynamics in different systems, same $\mathrm{Z}$ target nuclei and the selection of beam energies are very important for creating the same average angular momentum in the compound nucleus.

\section{References}

1. H. C. Britt and A. R. Quinton, Phys. Rev. 124, 877 (1964).

2. J. Galin, B. Gatty, D. Guirean, C. Rousset, V. C. Schlot-thauervoos and X. Tarrago, Phys. Rev. C 9, 1126 (1974).

3. T. Inamura, M. Ishilara, T. Fafuda, T. Shimoda and H. Hiruta, Phys. Lett. 68B, 51 (1977).

4. D. Singh, R. Ali, M. Afzal Ansari, M.H. Rashid, R. Guin, S.K. Das, Phys. Rev. C 79, 054601 (2009).

5. D. Singh, R. Ali, M. Afzal Ansari, B.S. Tomar, M.H. Rashid, R. Guin, S.K. Das, Phys. Rev. C 83, 054604 (2011).

6. R. Ali, D. Singh, M. Afzal Ansari, M.H. Rashid, R. Guin, S.K. Das, J. Phys. G: Nucl. Part. Phys. 37, 115101 (2010).

7. T. Udagawa, T. Tamura, Phys. Rev. Lett. 45, 1311 (1980).

8. J.R. Wu, I.Y. Lee, Phys. Rev. Lett. 45, 8 (1980).

9. J. Wilczynski et al., Nucl. Phys. A 373, 109 (1982).

10. J.P. Bondrof et al., Nucl. Phys. A 333, 285(1980).

11. R. Weiner, M. Westrom, Nucl. Phys. A 386, 282 (1977)

12. V. Zagrebaev, Y. Penionzhkevich, Prog. Part. Nucl. Phys. 35, 575 (1995).

13. D. Singh, R. Ali, M. Afzal Ansari, K. Surendra Babu, P. P. Singh, M.K. Sharma, B.P. Singh, R.K. Sinha, Rakesh Kumar, S. Muralithar, R.P. Singh, R.K. Bhowmik, Phys. Rev. C 81, 027602 (2010).

14. P. Pushpendra, Singh, B. P. Singh, M. K. Sharma U. Gupta, Rakesh Kumar, D. Singh, R. P. Singh, S. Muralithar, M. Afzal Ansari, R. Prasad and R. K. Bhowmik., Phys. Lett. B 671, 20 (2009).

15. R. K. Bhowmik, Inter-University Accelerator Centre (IUAC), New Delhi (unpublished).

16. RADWARE, the level scheme directory on http://radware.phy.ornl.gov/agsdir1.html. 